\title{
Potential impact of the interactions between Synechococcus and heterotrophic bacteria on oceanic carbon flow
}

\author{
Zenghu Zhang ${ }^{1}$, Lili Tang ${ }^{1}$, Richard B. Rivkin ${ }^{2}$,Yongyu Zhang ${ }^{1 *}$
}

\author{
${ }^{1}$ Qingdao Institute of Bioenergy and Bioprocess Technology, Chinese Academy of Sciences, \\ Qingdao, 266101, China \\ ${ }^{2}$ Department of Ocean Sciences, Memorial University of Newfoundland, St. John's \\ Newfoundland, A1C 5S7 Canada
}

*Corresponding author: E-mail: zhangyy@qibebt.ac.cn; Tel/Fax: +86-532-80662680

\begin{abstract}
Synechococcus accounts for a considerable proportion of marine primary production and has complex interactions with heterotrophic bacteria at sub-micrometer scales. However, the impact of the Synechococcus-bacteria interactions on marine carbon cycle is still unclear. Here, through laboratory experiments, we studied the potential role of the interactions between bacterial communities and two ecotypes of Synechococcus (i.e. PCC7002 and CCMP1334) in driving carbon flow. Synechococcus released abundant dissolved organic matters (DOM) during their growth. A large fraction of the DOM was rapidly utilized by heterotrophic bacteria and transformed into bacterial biomass. While partly since the DOM released from two Synechococcus had different chemical composition, which can be partially reflected by their differences in the fluorescent DOM (FDOM) components, the two Synechococcus reshaped the co-cultivated bacterial community into distinct community structure. Among the FDOM released by these two Synechococcus ecotypes, a portion of humic-like component was highly resistant to bacterial degradation and could accumulate in the co-culture system, suggesting that Synechococcus may directly produce recalcitrant DOM and contribute to the long-term sequestration of carbon in a dissolved form in the ocean. Meanwhile, the Synechococcus-bacteria interactions increased aggregate formation and particle sinking, suggesting that their interactions in the ocean may enhance the export of Synechococcus-derived organic carbon to deep sea through sinking process of biological pump.
\end{abstract}

Key words: Marine Synechococcus, bacteria, carbon export, carbon sequestration 